\title{
A Noise Reduction Method Based on Modified LMS Algorithm of Real time Speech Signals.
}

\author{
JAGADISH S. JAKATI ${ }^{1}$, SHRIDHAR S. KUNTOJI ${ }^{2}$ \\ ${ }^{1}$ Department of Electronics \& Communication Engg, \\ S. G. Balekundri Institute of Technology \\ Belagavi, VTU Research Scholar,Karanataka (State), INDIA \\ ${ }^{2}$ Department of Electronics \& Communication Engg, Basaveshwar Engineering College Bagalkot, \\ VTU Research Supervisor, Karanataka (State), INDIA
}

\begin{abstract}
In real time speech de-noising, adaptive filtering technique with variable length filters are used which is used to track the noise characteristics and through those characteristics the filter equations are selected The main features that attracted the use of the LMS algorithm are low computational complexity, proof of convergence in stationary environment. In this paper, modified LMS algorithm is proposed which is used to denoise real time speech signal. The proposed algorithm is made by combining general LMS algorithm with Diffusion least mean-square algorithm which increase the capabilities of adaptive filtering. The performance parameter calculation shows that the proposed algorithm is effective to de-noise speech signal. A full programming routine written in MATLAB software is provided for replications and further research applications.
\end{abstract}

Key-Words: - Speech enhancement. LMS, MATLAB, Modified LMS Algorithm, dB SNR, LLR. ISD.

Received: October 16, 2020. Revised: February 22, 2021. Accepted: March 15, 2021. Published: March 30, 2021.

\section{Introduction}

As Real time speech processing is a very important topic in today's digital media where the audio and video are stored into different digital data format such as .mp3 and avi etc. Noise can be added to the data (both audio and video) due to various reasons such as background noise, communication noise etc. It is difficult to eliminate those noises due to its nature at the time of capturing and transmitting of the signal. As a result it is essential to perform de-noising operation at the time of storing the media. Various algorithms are developed to de-noise speech signals. But most of those de-noised techniques used fixed filter where the characteristics of the filter are designed to detect and eliminate some specific types of noises. In real time, noises show random natures which are not possible to minimize through those kind of filter. This leads to the design of filter whose characteristics presents in the signal. Least Mean Square (LMS) [1] algorithm is one of the most used techniques in audio de-noising. In this paper, real time speech de-noising algorithm is proposed which tested on different type of noise

\section{Experimental Methods}

\subsection{Database}

This present work used the international standard NOIZEUS database for speech enhancement [17]. This database contains the speech recordings of 30 sentences from the IEEE sentence database produced by three male and three female speakers [17], [18]. Each speaker has spoken five sentences and recorded using Tucker Davis Technology (TDT) in a Speech Processing Lab at University Texas, Dallas, USA at a sampling frequency of $25 \mathrm{kHz}$ and later its down sampled to $8 \mathrm{kHz}$. Since most of the speech intelligibility application involve the processing signal frequency to a maximum of $10000 \mathrm{~Hz}$ to cover most important frequency 
components for signal intelligibility [17]. Each sentence is corrupted by eight different types of real-world noises (Babble, Restaurant, AWGN, Airport), and all the sentences include all of the phonemes in the American English language. The intermediate reference system (IRS) filters are used to obtain clean and noisy signals. The extracted noises segments are artificially added to the filleted clean speech signal in order to reach the desired SNR levels. A short description of the database is given in Table I. For more details regarding the database can be found from [17].

\section{Literature Survey}

Umasankar and Sairam [2] perform detailed analysis various noise reduction algorithm for various speech de-noising algorithm such as ANC, LMS and NLMS etc. For this comparisons the authors considers various types and amount of noises which are added to the actual noiseless speech and used to analyze different types of techniques. From the analysis, the authors found that the NLMS algorithm gives better de-noising capabilities than other algorithms.

Zhang and Tai [3] presented normalized LMS algorithm to get better de-noising performances. The modified algorithm is implemented on MATLAB software and compared the result to check the performance of the algorithm. The simulation result shows the presented algorithm can de-noise speech better than other algorithm.

Jebin and Ramya[4] presented distributed arithmetic architecture to implement LMS filter which is used to de-noise audio signal in real time. The whole architecture is designed at hardware level which is implemented on FPGA in the optimized way.

Deepaand Poongodi[5] used speech denoising algorithm to recognize real time speech signal. In this paper, Discrete Cosine Transform and LMS algorithm are used in this case. The Discrete Cosine Transform is used to remove some small amount of noise factor which are then used by the LMS algorithm to track and remove large noises. The comparison results shows that the proposed technique give better noise removing capability than existing techniques than other existing techniques for various types of noises.

V.A.Mane et al. [6] presented a comparison of de-noising of speech using LDM and LMS algorithm for automatic speech recognition system.
From the comparison results the authors obtained that the LMS algorithm can de-noise real time speech than LDM algorithm.

Raja and Shanmugam [7] used noise cancellation and speech enhancement techniques to implement algorithm for Hearing Impaired Person. A novel approach named VSSNDLMS algorithm is presented in this paper which is used to calculate minimum mean square error. It is useful to predict the noise which is then used by the filter in LMS algorithm. The result showing improvement in audio quality.

Shemi and Ali [8] used wavelet based technique to de-noise speech signal. The dual tree discrete wavelet transform with thresholding techniques are used for proper de-noising. The algorithm is implemented on MATLAB tool and the performance is compared with existing techniques using SNR and RMSE values.

George Baravdish et al. [9] presented Singular Value Decomposition (SVD) based audio de-noising with overlap-add method. Nonlinear PDE method with tapering model is used to decompose the audio signal which is then used to de-noise by SVD technique.

Dalei Wu et al. [10] use compressive sensing method to reduce noises present in speech and audio signal where Fourier transform and gradient descend line search algorithm are used combinely for this purpose. This method is suitable to minimize different types of White Gaussian noises.

ShaikhAkibShahriyar et al. [11] presented auto-encoder based on convolutional de-noising for speech de-noising. This system tries to separate noise features from the noisy signals which are then used to generate near clean signal. The Speech Command Dataset is used to check the performance of the algorithm.

K. Kirankumar et al. [12] uses hybrid technique to reduce external and internal noises present in any speech. To perform this, windowing method, Gaussian filter and Kalman filter are used combinely. This technique is used to clean speech signal with different types of noises and in all cases it gives better result with respect to existing.

A.M.Mutawa[13] presented speech enhancement in a single channel environment that analyzes both the magnitude and phase spectrum of the speech signal, The performance of the proposed method is compared with that 
of three conventional methods The experimental results show that the three objective measures (LLR, LSD, and STOI) of the proposed method gives better results over the conventional methods in four different noise Signal to Noise Ratio.

\section{Proposed Algorithm.}

Speech is assumed to be quasistationary and is analyzed framewise. We assume that at each time instance $n$, the clean speech signal $x_{t}(n)$ is degraded by additive noise $v_{t}(n)$, and the noisy signal is derived as $y_{t}(n)$.

In an additive noise model,

$y_{t}(n)=x_{t}(n)+v_{t}(n)$

where $y(n)$ is the noisy speech signal, $x(n)$ is the clean speech signal, and $\mathrm{v}(\mathrm{n})$ is the additive noise in the time domain. $\mathrm{t}$ is the frame number, $\mathrm{t}=1,2,3$, .... $\mathrm{N}$, and $\mathrm{N}$ is total number of frames.

In the frequency domain, Equation (1) becomes

$$
Y_{t}\left[\omega_{k}\right]=X_{t}\left[\omega_{k}\right]+V_{t}\left[\omega_{k}\right]
$$

where $Y_{t}\left[\omega_{k}\right], X_{t}\left[\omega_{k}\right]$ and $V_{t}\left[\omega_{k}\right]$ are Discrete Short Time Fourier Transform (DSTFT) representations of output, input and noise spectrum, respectively, and $k$ is the $k$ th discrete frequency. In this work, we assume that the harmonic frequencies and amplitudes are constant for a given length of speech signal using STFT.

The proposed speech de-noising algorithm is shown in the Figure 1 which consists of "Local Noise Generator", "Preprocessing" and "Modified LMS Algorithm"respectively. The speech signal is converted into finite number of frames by "Preprocessing". The frames are processed and denoised by "Modified LMS Algorithm" with the help

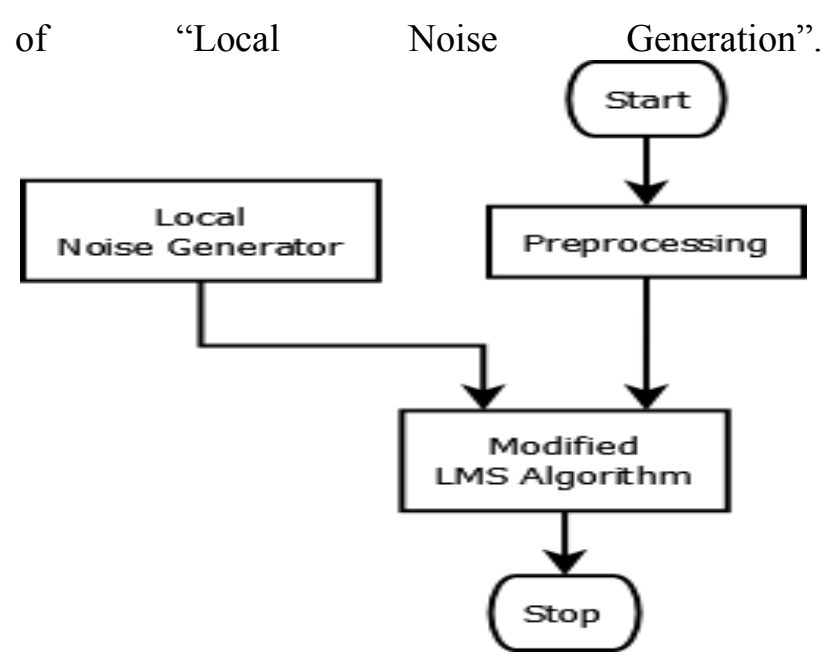

Fig. 1.: Proposed Speech De-noising Algorithm.

Preprocessing: The raw real time speech signal is processed to make it suitable for processing through digital algorithms. The Preprocessing performs windowing and filtering operations. The windowing method is used to divide the whole speech signals into a finite number of frames. To generate proper frames, Hamming window [14] is used in this implementation.

$\mathrm{wn}=\alpha+\beta \cos (2 \pi n N-1)$

Where, $\alpha=0.54$ and $\beta=0.46$

Also, the small amounts of noise are smoothening by the filtering operation pre sent in Pre-processing method. This helps the LMS algorithm to track the noise. Highpass all-pole filter is used for this purpose.

Local Noise Generator: The standard LMS algorithm requires some noise sources which are generated locally and used as initial noise characteristics to track the exact noise characteristics. Normally Gaussian noise is used to generate the local noise by the Local Noise Generator block. The equation is used to generate the Gaussian noise is given in Equation (1) as

local_noise $=\sin \left[\frac{1}{\left.1+\exp ^{- \text {wgn }\{\text { length(audio signal })}\right]}\right]$

Where, wgn is the white Gaussian noise function in MATLAB. 
Modified LMS Algorithm: The general LMS algorithm is shown in the Figure 2 where local noise source is needed to track the noise signal present in the real speech [5].The input signal $(\mathrm{Bk}+\mathrm{Nk})$ is delayed by one sample to de correlate the noise signal from the actual noisy signal which is represented (Nak+ Nek).

The internal detail of general LMS algorithm is shown in the Figure 3 where the coefficient of the de-noising filter is updated by the algorithm itself through feedback which gives better de-noising capabilities.

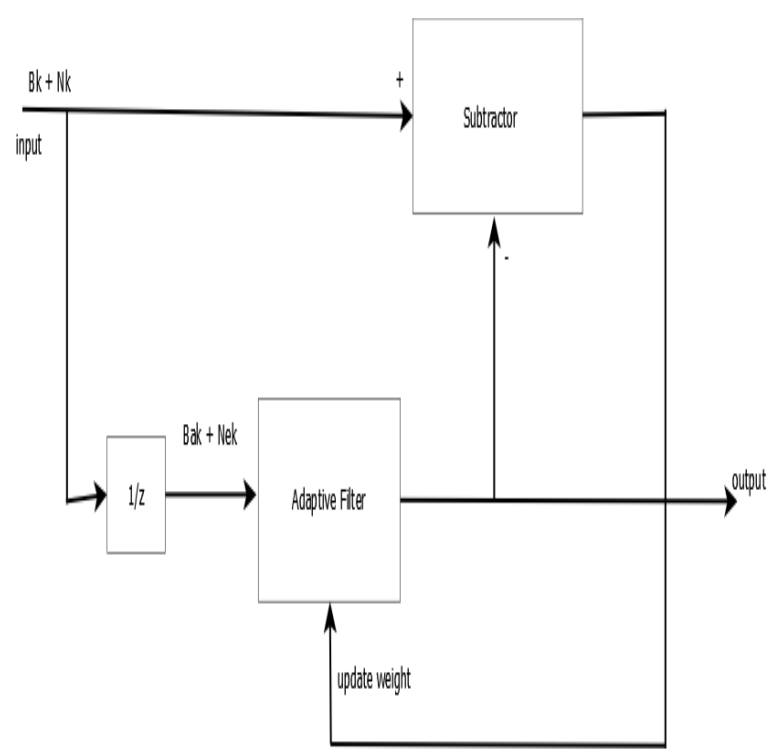

Fig. 2: General LMS Algorithm for Speech Denoising

TABLE I. DESCRIPTION OF THE SPEECH DATABASE.

\begin{tabular}{llc}
\hline $\begin{array}{c}\text { Segment } \\
\text { no }\end{array}$ & \multicolumn{1}{c}{ Parameter } & Values \\
\hline 1. & Total number of speech signals & 30 \\
2. & Total number of additive noises & 8 \\
3. & Total number of noisy signals & 960 samples: 30 clean signals \\
& & $\times 8$ types of noises \\
& & 4 SNRs \\
4. & SNR ranges & $0 \mathrm{~dB}, 5 \mathrm{~dB}, 10 \mathrm{~dB}$, and $15 \mathrm{~dB}$ \\
5. & Min duration of clean/noisy signals & $2.116 \mathrm{sec}$ \\
6 & Max duration of clean/noisy signals & $3.508 \mathrm{sec}$ \\
6. & Sampling frequency & $25000 \mathrm{~Hz}$ \\
7. & Downsampling frequency & $8000 \mathrm{~Hz}$
\end{tabular}




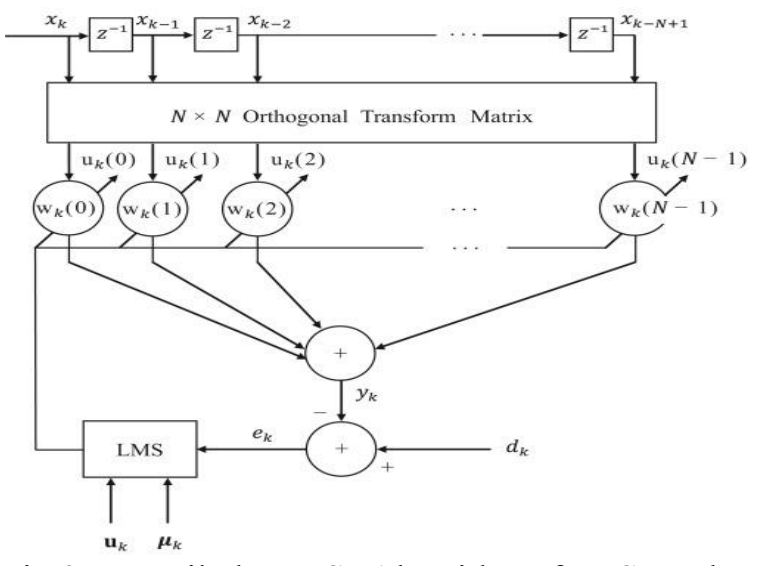

Fig.3: Detailed LMS Algorithm for Speech Denoising.

The weights of the filters presents in Figure 3 adjust at each frame by calculating the corresponding $\mathrm{N}_{\mathrm{k}}$ values using Equation (5)

$\widetilde{N_{k}}=\sum_{i=0}^{N-1} w_{k}(i) X_{k-i}$

The estimated error signal is then calculated using Equation (6) as

$e_{k}=y_{k}-\widetilde{n_{k}}$

To find optimal filter coefficients, the current input values which represent instantaneous gradient are multiplied by the learning coefficient $\mu$, which adjusts the filter coefficient values in LMS algorithm. The equation for this is given in

$\widetilde{w_{k+1}}=\widetilde{w_{k}}+2 \mu e_{k} \widetilde{X_{k}}$

To reduce the mean square error up to the maximum limit we must have to make the gradient value as zero which is not possible in practical case. In reality we can make this to nearer to zero by considering small steps for learning coefficients of $\mu$. In our implementation we consider the value of $\mu$ varies from $1 / \alpha^{4}$ to $1 / \alpha^{7}$ which is derived from eq. 8 as

$0<\mu<\frac{2}{\left\|\widetilde{X_{k}}\right\|^{2}}$

The regression vector $X_{k, n}$ and $Y_{k, n}$ are

$Y_{k, n}=X_{k, n}^{T} h+v_{k, n}$

Where, $\mathrm{v}_{\mathrm{k}, \mathrm{n}}$ is the noise

The intermediate estimation through previous estimation using the equation

$Z_{k, n}=W_{k, n-1}+\mu_{k} X_{k, n}\left(Y_{k, n}-X_{k, n}^{T} W_{k, n-1}\right)$
This helps to achieve accurate estimation factor which is the main reason for track and correct the noises present in the input speech frames.

\section{Performance Parameter}

The parameters are used to calculate the performance [9] of the proposed algorithm which is discussed briefly in this section.

Increment in Segmental SNR: In any speech signal, energies at are in non-stationery in the nature which fluctuate randomly. As a result, to get accurate SNR value each segment of frame is computed separately and combined to form segmental SNR. Thus the equation of segmented SNR is given in Equation (11) as

$\mathrm{SNR}_{\text {seg }}=\left(\frac{10}{1}\right) \sum_{\mathrm{i}=0}^{\mathrm{l}-1} \log _{10}\left(\frac{\sum_{\mathrm{n}=N_{\mathrm{i}}}^{\mathrm{N}_{\mathrm{i}}+\mathrm{N}-1} \mathrm{x}^{2}(\mathrm{n})}{\sum_{\mathrm{n}=\mathrm{N}_{\mathrm{i}}}^{\mathrm{N}_{\mathrm{i}}+\mathrm{N}(\mathrm{x}(\mathrm{n})-\widehat{\mathrm{x}}(\mathrm{n}))^{2}}}\right)$

Where, $\mathrm{N}$ is the frame length.

1 is the number of frames.

$\mathrm{x}(\mathrm{n})$ is the original noisy speech.

$\mathrm{x}^{\wedge}(\mathrm{n})$ is the processed speech signal.

Log Likelihood Ratio (LLR): Phase between the spectrum of clean speech and processed speech is calculated through LLR which gives the amount of

distortion added during processing. The equation is used to calculate LLR is shown in Equation (12) as

$D_{L L R}\left(a_{e}, a_{c}\right)=\log _{10}\left(\frac{a_{e} R_{c} a_{e}^{T}}{a_{c} R_{c} a_{c}^{T}}\right)$

Where, $a_{c}$ is the LPC vector of clean speech signal.

$a_{e}$ is the LPC vector of enhanced or processed speech signal. $R_{c}$ is the autocorrelation matrix of clean speech signal.

Itakura-Saito Spectral Distance (ISD): The difference between enhanced speech signal and clean speech signal in terms of the corresponding spectral envelope is known as Itakura-Saito spectral distance which is given in Equation (13). The standard value of ISD is always lesser than 100 .

$D_{I S D}\left(a_{e}, a_{c}\right)=\frac{G_{c}}{G_{e}}\left(\frac{a_{e} R_{c} a_{e}^{T}}{a_{c} R_{c} a_{c}^{T}}\right)+\log _{10}\left(\frac{G_{c}}{G_{e}}\right)-1$ 


\section{Performance Analysis}

To compare the performance of the proposed algorithm with existing with respect to different parameters. To perform these analyses, it is essential to calculate those parameters (dBSNR, LLR and ISD) for real time speech by inserting different type of noises. The performance of the proposed method is analyzed using four objective speech quality measures such as LLR, dBSNR, ItakuraSiato distance and it is compared with LMS Alogoritm. This present experiment revealed that the proposed method performed well in improving the quality of the speech signal over LMS Alogoritm and it is confirmed through the three objective measures namely, dBSNR, LLR, and ISD.For proper analysis, different level of noises $(0,5,10$ and $15 \mathrm{~dB})$ [9] is considered which are tabulated in in the Table II for different types of standard noises [9].

TABLE II. PERFORMANCE ANALYSIS OF THE MODIFIED ALOGIRITH.

\begin{tabular}{|c|c|c|c|c|c|c|}
\hline \multirow{2}{*}{ S. No. } & \multirow{2}{*}{ Noise Type } & \multirow{2}{*}{ Parameters } & \multicolumn{4}{|c|}{ Noise Level } \\
\hline & & & $0 \mathbf{d B}$ & $5 \mathrm{~dB}$ & $10 \mathrm{~dB}$ & $15 \mathrm{~dB}$ \\
\hline \multirow{5}{*}{1} & \multirow{5}{*}{ Airport } & Noisy SNR & 3.31 & 1.40 & 0.46 & 0.15 \\
\hline & & De-noised SNR & 0.01 & 1.68 & 2.62 & 2.91 \\
\hline & & dB SNR & 0.50 & 3.84 & 7.95 & 10.5 \\
\hline & & LLR & 1.32 & 1.12 & 0.98 & 0.77 \\
\hline & & ISD & 42.2 & 27.5 & 24.7 & 21.5 \\
\hline \multirow{5}{*}{2} & \multirow{5}{*}{ Babble } & Noisy SNR & 3.41 & 1.38 & $\mathbf{0 . 5 0}$ & $\overline{0.15}$ \\
\hline & & De-noised SNR & 0.19 & 1.80 & 2.60 & 2.92 \\
\hline & & dB SNR & 4.22 & 4.67 & 8.31 & 13.9 \\
\hline & & LLR & 1.36 & 1.11 & 0.93 & $\overline{0.76}$ \\
\hline & & ISD & 29.2 & 50.6 & 22.7 & 21.7 \\
\hline \multirow{5}{*}{3} & \multirow{5}{*}{ Restaurant } & Noisy SNR & 3.38 & 1.34 & 0.41 & 0.11 \\
\hline & & De-noised SNR & 0.14 & 1.84 & 2.68 & 2.94 \\
\hline & & dB SNR & -2.9 & 2.97 & 6.89 & 12.8 \\
\hline & & LLR & 1.37 & 1.08 & 0.91 & 0.79 \\
\hline & & ISD & 33.0 & 97.3 & 26.61 & 50.5 \\
\hline \multirow{5}{*}{4} & \multirow{5}{*}{ AWGN } & Noisy SNR & 3.00 & 1.17 & 0.44 & 0.13 \\
\hline & & De-noised SNR & 3.22 & 0.11 & 1.77 & 2.62 \\
\hline & & dB SNR & 3.22 & 4.99 & 8.10 & 11.9 \\
\hline & & LLR & 2.51 & 2.21 & 1.85 & 1.60 \\
\hline & & ISD & 73.2 & 76.6 & 117.6 & 131. \\
\hline
\end{tabular}




\section{Simulation results}

This section presents the experimental results of the present work. All the experiments were performed on the NOIZEUS speech corpus database to analyze the performance of the proposed speech enhancement method and compare denoise speech signal. This database has been used by several researchers for speech enhancement applications and its gender-matched database, and it has 30 IEEE sentences. One of the speech sample (Train) at $10 \mathrm{~dB}$ SNR is excluded from this work for analysis.

Initially, the speech samples from the database are framed using a hamming window method with a window length of $25 \mathrm{~ms}$ over the $40 \%$ overlapping between the frames. This windowing is applied to all the speech samples in the database. Later, the speech samples are added with an additive Gaussian noise of different SNR of the same window length of original speech signals. Then, the STFT is applied to the noisy speech signals and extracted its magnitude and phase spectrum values.

Finally, the speech signals are reconstructed to derive the time domain speech signals using a two-stage approach. Later, four different objective measures are computed from the original (clean) speech signal and enhanced speech signal for performance comparison over each frame. The time series plot of the original (clean) and enhanced speech signal over four different SNRs.. This plot represents the signal variations over the 15000 samples for different SNRs.

The spectral power distribution over frequency for a speech signal with airport noise at $0 \mathrm{~dB}, 5$ $\mathrm{dB}, 10 \mathrm{~dB}$, and $15 \mathrm{~dB}$. From the results, it indicates that the proposed speech enhancement method gives the lowest value of LLR over the other three methods. In specific, the performance of the proposed method has shown the improvement in speech enhancement process over conventional methods. Also, the average value of LLR close to zero (enhanced signal is almost similar to clean signal) when the noise SNR increases. The LLR variations at different noise levels $(5 \mathrm{~dB}, 10 \mathrm{~dB}$ and $15 \mathrm{~dB})$. The experimental results confirm that the proposed method achieves lower LLRvalues than LMS algorithm speech enhancement methods for most types of noise.

The proposed method is derived from the modified LMS algorithm, with modification in frequency spectrum computation. Firstly, the proposed method analyzes the speech signal of fixed frame duration, it is also important to analyze the performance of the proposed method with different frame durations. Secondly, the proposed method should be tested with other open-source and international standard speech corpus to validate its efficiency in speech enhancement process. Thirdly, the proposed method is evaluated only through objective measures, but, it is also important to analyze the performance of the proposed system using subjective measures and with a combination of subjective and objective measures. Lastly, the proposed method is evaluated through only a selected and most popular objective measures for analyzing its performance

The Actual Signal, Noisy Signal and De-noised Signals are plotted with respect to time domain in the Figure 4 from which it can be seen that the characteristics of the de-noised signal is almost similar to the characteristics of the actual signal, but the amplitude of the de-noised signal is lesser than actual signal which is corrected by applying correct gain factor at the final output stage to get proper amplitude in terms of volume.

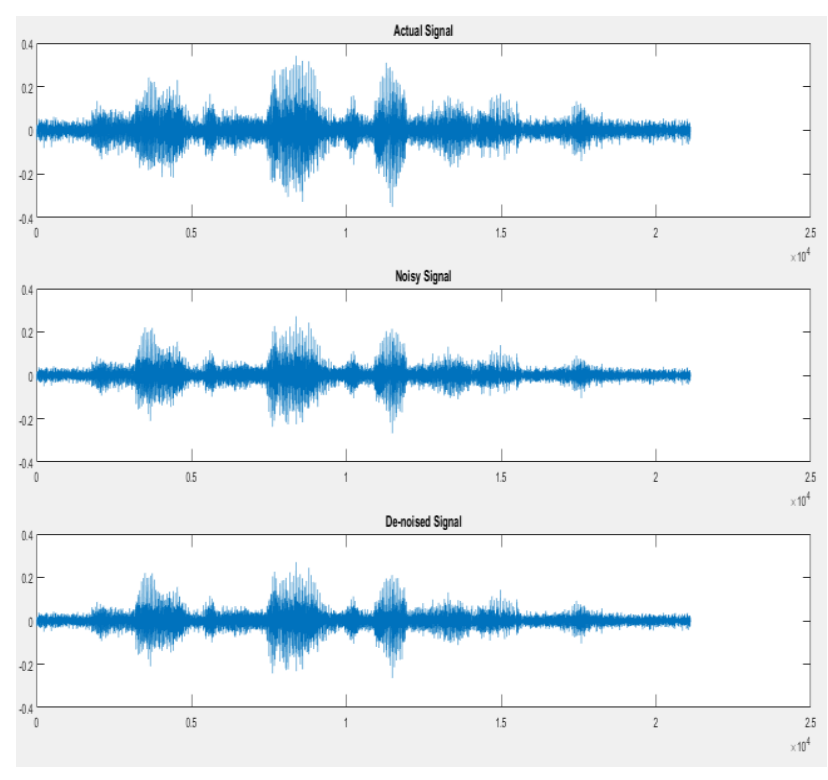

Fig. 5: Signal Comparisons in Time Domain.

The frequency components presents in the True Speech Signal, Noisy Speech Signal and De-noised Speech Signals respectively are shown in the Figure 5 with the help of Spectrum Analyzer present in MATLAB [8] software. From Figure 6, it can be seen that the spectrum of the de-noised speech signal is very near to the spectrum of the actual speech signal which proves that the proposed algorithm is efficient to track and remove noises from real time speech signals. 


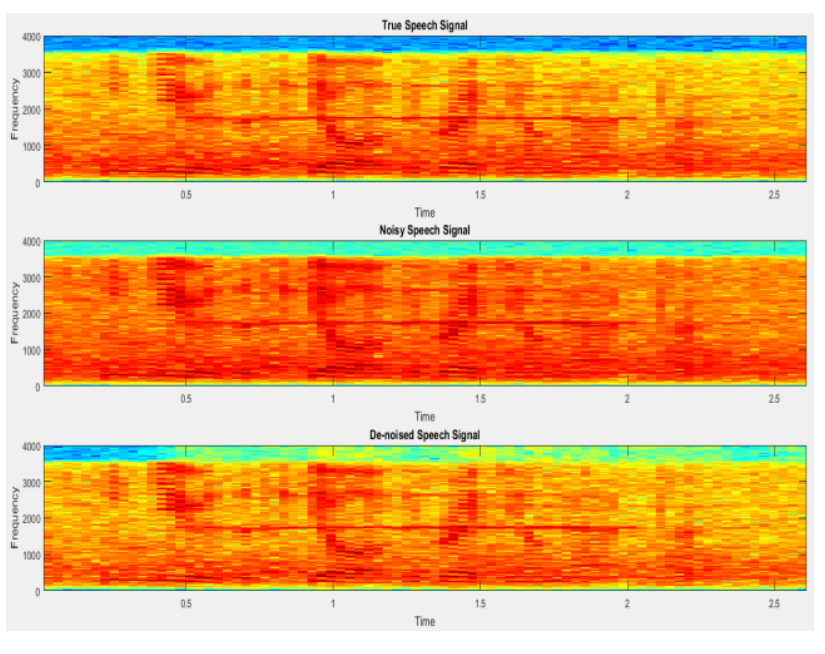

Fig. 6: Signal Comparisons in frequency domain.

\section{Conclusion}

In this paper modified LMS algorithm is proposed and the performances of the adaptive filter using MLMS algorithm is discussed. The proposed algorithm that adaptive filters operated in frequency domain and time domain input signals and this algorithm to get good de-noise algorithm the existing LMS algorithm is modified with the help of diffusion least mean-square algorithm. This present experiment revealed that the proposed method performed well in improving the quality of the speech signal over LMS Algorithm and it is confirmed through the three objective measures namely, dBSNR, LLR, and ISD. Future work should focus on analyzing the performance of the proposed method under different operating conditions, including different noise environments performance measures by combining the objective and subjective measures to evaluate the performance of the proposed algorithm.

\section{Acknowledgment}

Authors would like to sincerely thank Dr. S. S. Injaganeri, Principal, Basaveshwar Engineering College, Bagalkot, Karnataka and Dr. Sidramappa V.Itti, Principal, S. G. Balekundri Institute of Technology, Belagavi, Karnataka and dear staff member of BEC, Bagalkot and SGBIT, Belagavi for their kind support in carrying out this research work.

\section{References:}

[1] Thomas F. Quatieri, "Discrete-Time Speech Signal Processing: Principles and Practice",Pearson Education, 1st Edition, 2003.

[2] Ch. D. Umasankar and M SatyaSairam, "Performance Analysis of LMS, NLMS Adaptive Algorithms for Speech Enhancement inNoisy Environment", International Journal of Innovative Technology and Exploring Engineering, Vol. 9, Issue. 4, pp.2330-2333, February 2020.

[3] Jiashu Zhang and Heng-Ming Tai, "Adaptive Noise Cancellation Algorithm forSpeech Processing", 33rd Annual Conference of the IEEE Industrial Electronics Society, pp. 24892492, 2007, Taiwan.

[4] Jebin Roy and R. Ramya, "Low Power and Low Area Adaptive FIR Filter based on Arithmetic Algorithm" International Journal of Scientific and Research Publications, Volume 4, Issue 3, 2014.

[5] Deepa .D andPoongodi .C, "Speech Enhancement Using Dual TransformNormalized LMS Algorithm for Speech Recognition Application", International Journal of Advance Research, Ideas and Innovations in Technology, pp. 729-735,2017.

[6] V.A.Mane, K.P.Paradeshi, S.A.Harage and M.S.Ingavale, "Comparison Of LDM and LMS for an Application of a Speech", International Journal on Signal Processing, Vol. 5, Issue 4, pp. 130-141, 2011.

[7] M.A.Raja and A.Shanmugam, "Noise Cancellation and Speech Enhancement for Hearing Impaired Person", International Journal of Advanced Research in Computer Science, Vol. 4, No. 4, pp.8-12, 2013.

[8] Shemi P. M. and Ali M. A, "An Improved Method of Audio Denoising Based on Wavelet Transform", IEEE International Conference on Power, Instrumentation, Control and Computing, pp. 1-6, 2015.

[9] George Baravdish, Gianpaolo Evangelista, OlofSvensson and FatenSofya, "PDE-SVD Based Audio Denoising”, 5th IEEE International Symposium on Communications, Control and Signal Processing, pp. 1-6, 2012.

[10] Dalei Wu, Wei-Ping Zhu and M.N.S. Swamy, "A Compressive Sensing Method for Noise Reduction of Speech and Audio SIgnals", 54th IEEE International Midwest Symposium on Circuits and Systems, pp. 1-4, 2011.

[11] ShaikhAkibShahriyar, M. A. H. Akhand, N, Siddique and T. Shimamura, "Speech Enhancement Using Convolutional 
DenoisingAutocoder", IEEE International Conference on Electrical, Computer and Communication Engineering, pp. 1-5, 2019.

[12] K. Kirankumar, M. Suneel and P. Surendrakumar, "Hybrid Technique for Denoising Multi Environment Noise in Speech Processing", 9th IEEE International Conference on Computing, Communication and Networking Technologies, pp. 1-7, 2018,

[13] A. M. Mutawa, "Single Channel Speech Enhancement using a Complex Spectrum Method, "International Journal of Circuits, Systems and Signal Processing, pp. 667-677, Volume 13, 2019.

[14] A. M. Mutawa, "Improving Patient Voice Intelligibility by using a Euclidian Distancebased Approach to Improve Voice Assistant Accuracy", International Journal of Circuits, Systems and Signal Processing, pp. 329-339, Volume 14, 2020

[15] Jagadish S.Jakati and Shridhar S.Kuntoji, "Speech Enhancement Using Novel TimeFrequency Analysis Techniques: A Survey on Comparison.", International Journal of Advanced Trends in Computer Science and Engineering, Vol. 9, No. 4, pp. 42294234,2020.

[16] Jagadish S.Jakati and Shridhar S.Kuntoji, "Efficient Speech De-noising Algorithm using Multi-levelDiscrete Wavelet Transform and Thresholding",International Journal of Emerging Trends in Engineering Research, Vol. 8, No. 6, pp.2472-2480,2020.

[17] Y. Hu and P. Loizou, "Subjective evaluation and comparison of speech enhancement algorithms," Speech Commun., vol. 49, pp. 588-601, Aug. 2007.

[18] IEEE Subcommittee, "IEEE recommended practice for speech quality measurements," IEEE Trans. Audio Electroacoustics, vol. 17, pp. 225-246, Jun. 1969.

[19] Dr.(Mrs) S.D.Apte, Shridhar. " Speech Enhancement in Hearing Aids using Conjugate Symmetry Property of Short Time Fourier Transform" International Journal of Recent Trends in Engineering, Academy publishers, Finland, Nov 2009, Vol 2, No 1-6, pp 346 -351.

[20] Dr.(Mrs) S.D.Apte, Shridhar. " Speech Enhancement in Hearing Aids using Conjugate Symmetry of DFT and SNR-Perception Models " International Journal of Computer Applications (0975-8887)". Computer Society of America Publishers, USA. 2010 Volume 1No.21. pp 43-51

\section{Contribution of individual authors to the creation of a scientific article (ghostwriting policy)}

\author{
Jagadish S.Jakati: Has conceived the presented \\ idea, methodology, implementation, writing, \\ reviewing, and editing.
}

Follow: www.wseas.org/multimedia/contributorrole-instruction.pdf

\section{Creative Commons Attribution License 4.0(Attribution 4.0 International ,CC BY 4.0)}

This article is published under the terms of the Creative Commons Attribution License 4.0 https://creativecommons.org/licenses/by/4.0/deed.en US 